\title{
Still Face Image Object Detection using EV-Jones Algorithm
}

\author{
Gogineni Rajesh Chandra \\ Research Scholar, Department of \\ Computer Science \\ Acharya Nagarjuna University, \\ Nagarjuna Nagar, Guntur, AP, \\ India
}

\author{
Kolasani Ramchand H. Rao \\ $\mathrm{PhD}$ \\ Professor, \\ Department of Computer Science, \\ ASN Degree College, Tenali, AP, \\ India
}

\author{
V. V. Jaya Rama Krishnaiah \\ $\mathrm{PhD}$ \\ Associate Professor, Department \\ of Computer Science and \\ Engineering, ASN Women's \\ Engineering College, Tenali, AP, \\ India
}

\begin{abstract}
Many applications are developed for identification of biometric, classification, cryptography information, identification of forensic, control data access, border surveillance using human face and interaction of human etc. In our work, we have developed Vision based MATLAB tool for identification of various parts of face in human like eyes, ear and nose etc. this tool is developed based on EV-JONES detection of face algorithm. When this algorithm is applied each of the threshold values of face parts are identified and successfully detection based on various types of images which contain one or more objects related to faces in it.
\end{abstract}

\section{Keywords}

Image processing, face detection, learning, Boosting

\section{INTRODUCTION}

Recognition of face is the ongoing research area in the pasted and present. Various parts of face are detected based on object images and videos from various types of sources. Recognition and identification of face system has two operations to perform: identification and verification.

\subsection{Technique for Detection of Face}

Techniques for detection of face is given below

\section{a. Traditional}

Algorithms which are traditional will identify the features of facial and extract only the important and landmarks in the image which is subjected to face only. Some popular recognition of face is PCA, model of Markov hidden model, subspace multiline learning using discriminant analysis and tensor etc.

\section{b. Three-Dimensional Recognition}

This method used sensor of 3D in capturing the image information and its face shapes. By this image it is each to identify and distinctive the surface of face features such as sockets eye and counter of noise and chin.

\section{c. Skin texture analysis}

Using this technique spot of skin and pattern of particular apparent of a person is known and is called as texture skin analysis.

\section{d. Thermal cameras}

Cameras of special are used in shape detection of head and will discard the accessories related to unnecessary like glass, hats and makeup

\section{LITERATURE REVIEW}

Many developments in the face detection algorithms has been done in past years. Multi resolution rule method was implemented by G. Yang[1]. This method used the structural nature of face for detection. Feature based method uses The color of skin[4], features of facial[2] and multiple combined features[6] of face to find effective accuracy at high speed. An uniform and steady image scaled method of template is used for matching. Some predefined templates was used[7] with a templates of deformable[8] which incorporated the complete template based on predefined structure based on learning. A method of appearance will speed the process of detection very fast and accurately with adaptive results which can distinguish face with non-face under environmental conditions.

\section{INTRODUCTION TO VIOLA-JONES OBJECT DETECTION FRAMEWORK}

The EViola-Jones object detection framework is extended version for detection accurate object detection framework which proposes competitive detection of object rates in real time where the basic version was proposed by Paul Viola and Michael Jones[9][10] . The early proposed algorithm only detects particular class of object, this motivation has made us to solve primary problem in identifying the face shape detection of objects.

\section{Extended Viola Jones Approach}

It is a fast and accurate method of detection of image object which has been devised from Jones in the year 2014.

This approach is most commonly used in smart phone, camera phones with high perimeters of security and is listed as so on. Speed of computational will be increase compared to Haar, A process of Machine Adaboost learning is used in detection of face within milliseconds. Initially each of the pixel vales of image with gray scale and black are accumulated, these values are subtracted from the whole of the white box. The results are gained and compared with the threshold defined to check the criterior of the required is met based on the features and identification [13].

In this approach objects are detected through these four key concepts.

1. Features of rectangle shape - named Haar-like features.

2. Image Integral for fast detection of image

3. AdaBoost method of Machine learning 
4. Combined classifier and Cascade for combine features efficiently

\section{Features of Haar}

Feature of Haar is a process of detecting the light and black portion of image. It is a form of rectangle shape computation process of detection of face. Based on the shade of color and near node or contour forehead formed [14]. Sometimes commonly known as features of Haar are

Two, Three and Four rectangle features

\section{Image Integral}

These are known as tables of image areas. Image integral is a process of quick felicitate detection of features[14]. Image integral is a process of outline the original picture image from the original image.

\section{Adaboost machine learning method}

It uses an important concept of Bagging that is procedure for combining different classifiers constructed using the same data set. It is an acronym for bootstrap aggregating, a motivation of combining classifiers is to improve an unstable classifier and an unstable classifier is one where a small change in the learning set/classification parameters produces a large change in the classifier.

AdaBoost method algorithm will help us to select tiny features from the face which facilitates easy and fast computation. As Like other algorithms and methods AdaBoost will desire region of portion of object which discards the object background unnecessary. The model of working is interpreted using Neural network model.

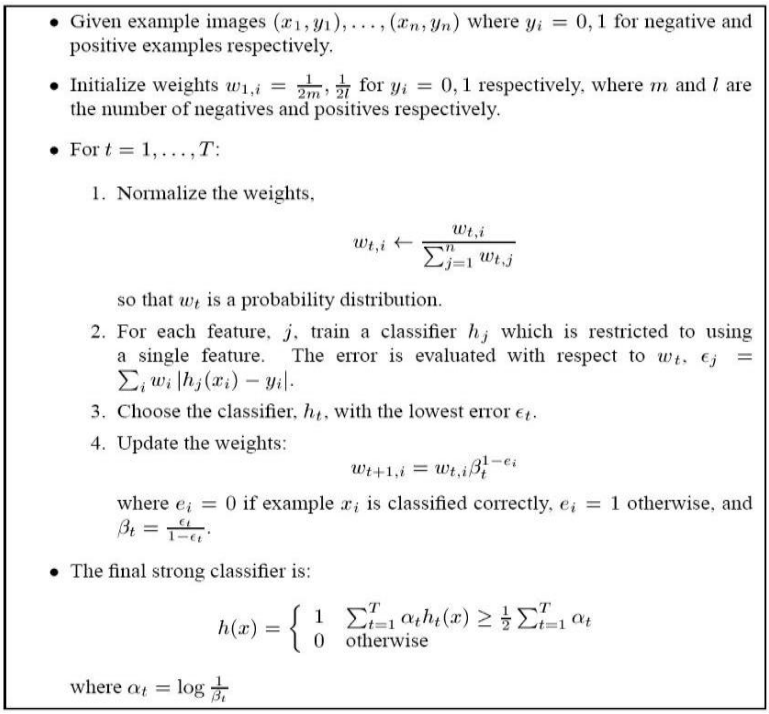

Figure 1: AdaBoost method of Learning algorithm

BoostADA method of learning is an efficient and quickly process for identifying the required data. The data which is identified is classified into certain classifiers. Each of the classifier contains tiny features of face. This is commonly done for identifying the pattern of detection. The proposed methods has high rate of accuracy and speed, but it has $1 \%$ of false detection as it requires some amount of time in training.

\section{Cascade based classifier}

The algorithm proposed will eliminate the candidate face quickly based on the stages of cascade. In each stage the eliminator cascade the candidate object image as stricter in each of the stage, which makes the candidates to pass the cascade stage strictly. A candidate object image will be exited if it fails to detect a face in each of the stage. If the object image of candidates passes in certain stage then only detected of face is passed. This process continuous as shown from Figure 2.

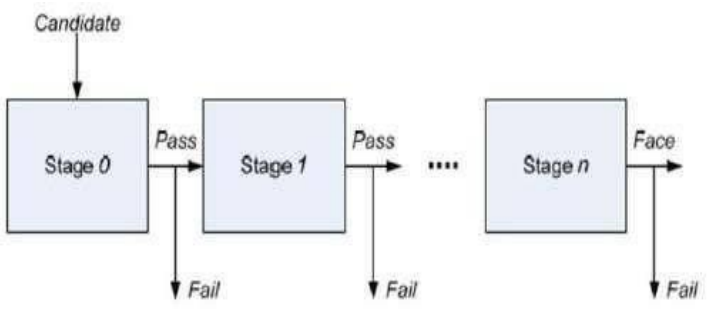

Figure 2: Stage wise cascade : Each of the object image portion has to pass through the phase for detection of face

\section{METHODOLOGY AND RESULTS}

In this work, MATLAB in-built APIs are used to detect face, upper body, nose, mouth and eyes etc. Viola-Jones face detection algorithm, the computer vision system toolbox contains vision. Cascade Object Detector System object which detects objects based on above mentioned algorithm. MATLAB 2014 is used in this work, which has Computer vision system toolbox in the default tool box list.This complete work is divided into six segments in which various parts of the face is detected.
A. Detection of Face
B. Body Upper Part Detection
C. Eye Single Detection
D. Detection of Mouth
E. Pair of Eye Detection
F. Detection of Nose

The details of these segments are as follows:

\section{A. Detection Of Face}

The object face detection can be done using Cascade vision Detector method which has face as default object. In this work face detection is performed on various types of images which include images with one, two, three and ten different faces as objects.Figures 3, 4, 5 and 6 shows images with one, two, three and ten faces respectively as an image object.

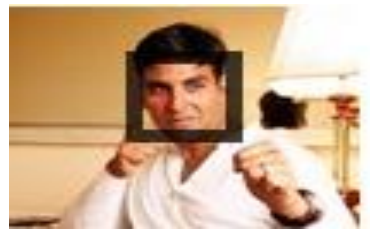

Figure 3: Detection of Face in 1 object

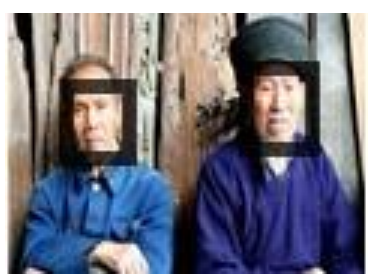

Figure 4: Detection of Face in 2 objects

The area of rectangle shows the face detection and 
identification related areas in image object.

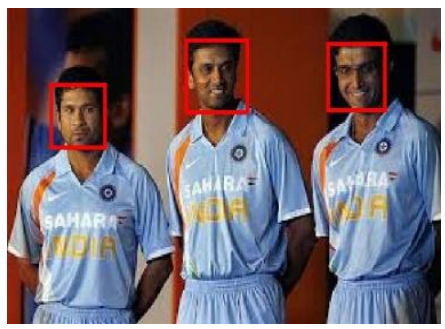

Figure 5: Detection of face in 3 object images

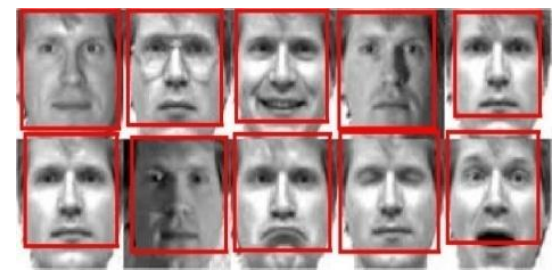

Figure 6 Detection of Face in Ten objects

It is observed from the figures that portion of face in each object image is detected successful and identified for various types of images as shown in figure 6 , which gives the reference of 10 faces shown in certain image and identified successfully.

\section{B. Body Upper Part Detection}

Figure 7, Figure 8, Figure 9 and Figure 10 below shows the body upper part image of the objects in the 1,2,3 and 4 respectively.

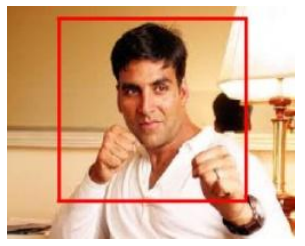

Figure 7: Detection of Upper body detection in 1 object

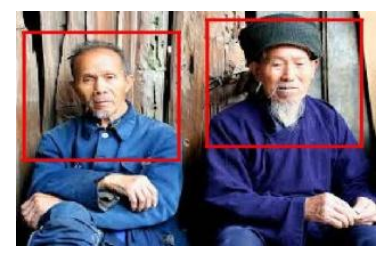

Figure 8: Detection of Upper body detection in 2 object

It has been verified from figures that upper portion of body parts are clearly visible and detected from 1 and 2 objects. The rectangle shape portion clearly shows the dis similarities among the faces in upper body.

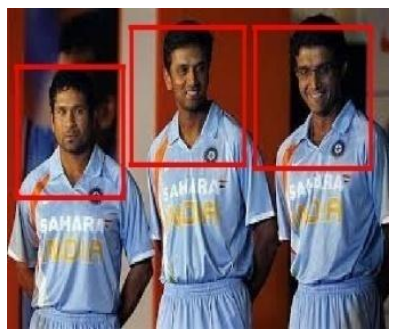

Figure 9: Detection of Upper body parts within three objects

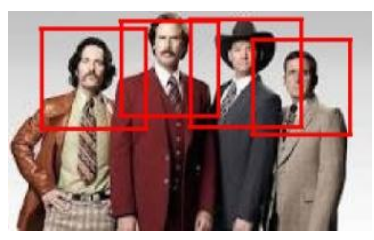

Figure 10: Detection of Upper body $n$ with 4 objects

In figure 10 and 9 showed above related to upper body part from 3 or 4 various types of objects. It has been observed that the method proposed by us will detect and identify the portion of upper body part successfully from various types of objects.

\section{EYE-PAIR SINGLE DETECTION}

In this method single eye of one and two objects is detected which is shown in figure 11 and 12 . Both the eyes are separately differentiated through two rectangles. Right and left eye is shown with different rectangles.

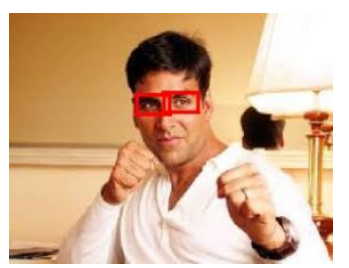

Figure 11: Detection of eyes Single in one object

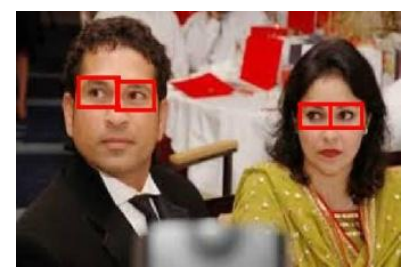

Figure 12: Detection of Single eye with in two image objects

\section{Detection Of Mouth}

Different types of mouth objects in various shapes are detected as shown in figure 13,14 and 15

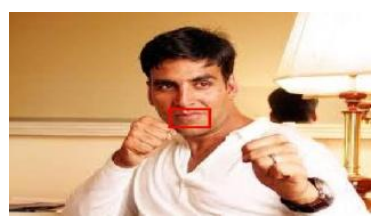

Figure 13: Detection of Mouth in one objects

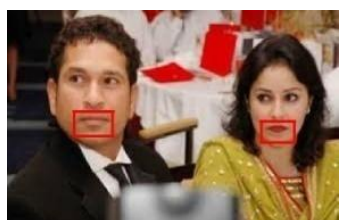

Figure 14: Detection of Mouth in two objects

Figure 13 and 14 above shows that detection of mouth images with one or more objects and its properties are respectively identified. 


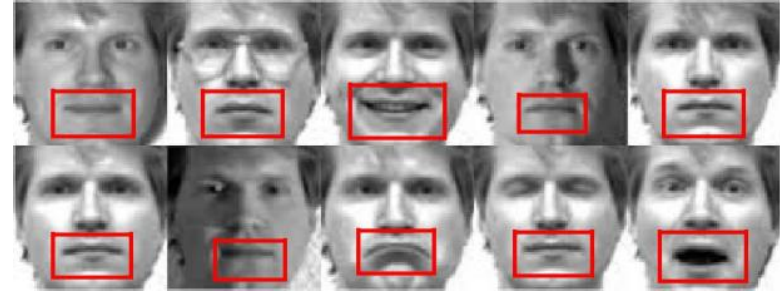

Figure 15: Detection of Mouth using ten image objects

It has been shown from results that properties of mouth detection in images of one, two and ten object images are done well. From figure 15, we have chosen 10 various types of faces ,our method has successfully detected the structured difference among the faces.

\section{E. DETECTION OF PAIR-EYE IMAGE}

Figure 16 below shows the pair of eyes detection using the proposed method. Image Zoomed of pair of eyes is shown below in figure 17 ,

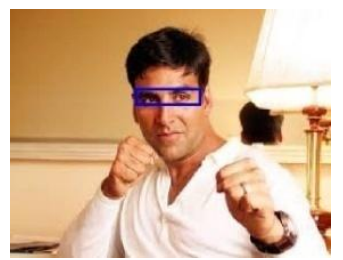

Figure 16: Detection of Pair of Eye

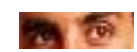

Figure 17: Figure 16 Shows Eye Zoomed image

\section{F. Detection Of Nose}

This section is used to identify image nose from various pictures, threshold is applied in this method, the threshold value is 4 , it can also be varied in between 3 to 18 in various types of gestures of face image. Figures 18, 19 and 20 shown below gives us the detection of Nose in one, two and nearly 10 object images respectively.

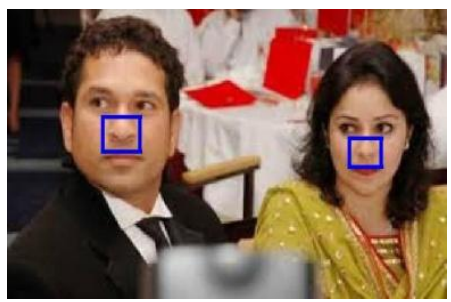

Figure 18: Detection of Image Nose in one object

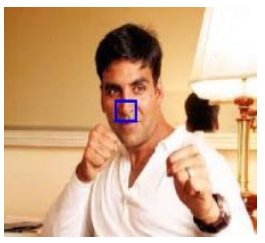

Figure 19: Detection of Image Nose in Two objects

From figure 19, 2 objects in certain images have postures of different; these parts are also clearly identified

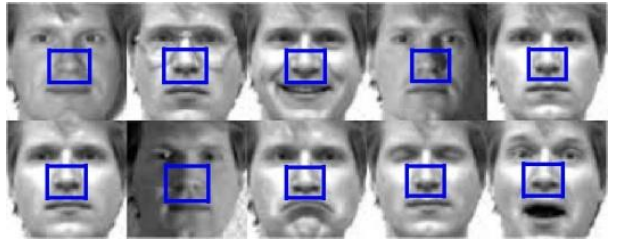

Figure 20: Detection of Image nose in 10 objects

It is shown from the results that object nose is identified clearly in various types of picture images.

\section{CONCLUSION}

Our work is based on image processing features which are implemented in MATLAB which is used for identifying the human parts in face. In our work V-Jones algorithm is used for detection of face. The algorithm implemented in MATLAB has inbuilt function based on vision and Detector cascade package. Mouth, eye, upper body, nose and other portion of human image is detected from various postures of objects in faces. This method develop will find all the parts in human face successfully for various types of images and pictures.

\section{REFERENCES}

[1] G. Yang and T. S. Huang,"Human Face Detection in Complex Background", Pattern Recognition, vol. 27, no. 1, pp. 53-63, 1994.

[2] I. Craw, D. Tock, and A. Bennett, "Finding Face Features" Proc. Second European Conf. Computer Vision, pp. 92-96, 1992

[3] T.K. Leung, M.C. Burl, and P. Perona, "Finding Faces in Cluttered Scenes Using Random Labeled Graph Matching", Proc. Fifth IEEE Int'l Conf. Computer Vision, pp. 637-644,1995.

[4] K.C. Yow and R. Cipolla,"Feature-Based Human Face Detection", Image and Vision Computing, vol. 15, no. 9, pp. 713-735, 1997.

[5] J. Yang and A. Waibel, "A Real-Time Face Tracker", Proc. Third Workshop Applications of Computer Vision, pp. 142147, 1996.

[6] S. McKenna, S. Gong, and Y. Raja, "Modelling Facial Colour and Identity with Gaussian Mixtures", Pattern Recognition, vol. 31, no. 12, pp. 1883-1892, 1998

[7] R. Kjeldsen and J. Kender, "Finding Skin in Color Images",Proc. Second Int'l Conf. Automatic Face and Gesture Recognition, pp. 312- 317, 1996.

[8] I. Craw, D. Tock, and A. Bennett, "Finding Face Features", Proc. Second European Conf. Computer Vision, pp. 92-96,1992

[9] A. Lanitis, C.J. Taylor, and T.F. Cootes, "An Automatic Face Identification System Using Flexible Appearance Models", Image and Vision Computing, vol. 13, no. 5, pp. 393-401,1995.

[10] Hsu, Rein-Lien, Mohamed Abdel-Mottaleb, and Anil K. Jain. "Face detection in color images." Pattern Analysis and Machine Intelligence", IEEE Transactions on 24.5 (2002):696-706.

[11] A.S. Georghiades, P.N. Belhumeur, D.J. Kriegman, "From few to many: illumination cone models for face recognition under variable lighting and pose", IEEE Trans. Pattern Anal. Mach. Intell. 23 (6) (2001) 643-660. 
[12] Mayank Chauha and Mukesh Sakle. "Study \& Analysis of Different Face Detection Techniques" International Journal of Computer Science and Information Technologies, Vol. 5 (2), 2014, 1615-1618.

[13] A. Lanitis, C.J. Taylor, and T.F. Cootes, "An Automatic Face Identification System Using Flexible Appearance Models", Image and Vision Computing, vol. 13, no. 5, pp. 393-401, 1995.

[14] H. Rowley, S. Baluja, and T. Kanade, "Neural NetworkBased Face Detection", IEEE Trans. Pattern Analysis and Machine Intelligence, vol. 20, no. 1, pp. 2338, Jan. 1998.

[15] S. McKenna, S. Gong, and Y. Raja, "Modelling Facial Colour and Identity with Gaussian Mixtures", Pattern 